\title{
On Tong and Huo in the Practice of Health Qigong
}

\author{
Wu Xu ${ }^{1}$ \\ ${ }^{1}$ School of Physical Education, Neijiang Normal University, Neijiang Sichuan, 641112
}

Keywords: Health Qigong, Tong, Huo

\begin{abstract}
The Chinese term Tong and Huo are two important indivisible elements in Chinese Health Qigong. The term Tong is the body of Tao, which refers to the smoothness, fluency and coherence of the movements, breath, mind, spirit and the whole body and also the unity of heaven, mankind and nature, etc.. The term Huo is the application of Tao, which refers to the flexibility and agility of the movements, contents, forms, thought and adjustment of body according to different situations. Tong and Huo have made up the relations of dialectical unity, which is a significant way of regime and the cultural foundation of the inheritance, promotion and introduction of Health Qigong.
\end{abstract}

\section{Introduction}

Health Qigong, as a comprehensive regimen and combination of body movements, respiratory regulation and psychological adjustment, is an important component of Chinese culture and traditional sports.

Health Qigong has a profound and multi-cultural foundation like a big tree with wide spreading branches and luxuriant foliage, rooted in the Chinese land of culture, absorbing the nutrition of the Chinese philosophy and cultural thought and the core of Chinese medicine and aesthetics. Therefore, Health Qigong is a regimen of unity of body and soul, self-cultivation internally and externally and unity of mankind, nature and society. To have a better practice of Health Qigong, the practitioners should comprehend the philosophy and theory of different exercises, practice flexibly, naturally according to the regulations, finally try to reach the unity of mental adjustment, respiratory regulation, physical adaptation and enjoy the unity of nature and mankind.

\section{Tong-the Body of Tao}

Tong, unobstructed, in Chinese dictionary of Cihai, has many meanings. One refers to smoothness and fluency, and another means having a good knowledge of or understanding something. Based on the interpretation of the Taoist thought, it has the following three meanings:

Firstly, it means that to follow the regulations of the event, things will go smoothly. In practicing Health Qigong, the practitioners should find out and conclude the regulations. Just as a Chinese forefather said, "if the form or posture is not correct, the respiratory can not be in regulation, then the mind and attention can not be focused, eventually, the spirit and energy must be in disorder or dispersed", which can also be the conclusion of Health Qigong about the form or posture. The requirement here is to have a correct form and posture so that the bones and muscles will be relaxed and easy and the meridians and respiratory will be regulated. It is very important for practitioners to find the right regulations of the exercises.

The second meaning of Tong is the unity of mankind, heaven and nature. Mankind is an inseparable component of nature, which has shown the following three relations: human's physical periods are related to the change of nature; the change of human body follows the law of nature; the human body and nature share the same components and substances.

To realize the unity of mankind, heaven and nature, the practitioners should try to learn and know more about the origins and principles of the exercises and the physiological functions of the body, apply the theories of Yin and Yang, Five Elements, the meridians, the state of internal organs, Holism in practice and experience and taste the harmonious and unitive attachment of human body, spirit and nature through the exercises of Health Qigong, through which Tong is realized.

The third meaning of Tong can be simply interpreted as achieving mastery through a 
comprehensive creatively study and apply of Health Qigong, which asks the practitioners to read more thesis and professional documents, broaden their mind and do more practice and conclusions.

\section{Huo- the Application of Tao}

The Chinese term Huo means to be alive, active, flowing, flexible, agile, changeful and liberal, and sometimes it also means activities. Huo in Health Qigong is the distillation of Tong. Without the comprehension of the theory and practice of the movements, postures, contents and thought of Health Qigong, the practice of the exercises will be like a drought spring.

To achieve Huo, the first demand is the flexibility and agility of movements and postures, namely motor coordination, which is the core of all the exercises of Health Qigong, such as shrinking and straightening, stretch and release, virtual and real, open and close, square and round, roll and release, light and heavy, hard and soft, slow and fast, upward and downward, internal and external, left and right, big and small, advance and retreat.

Motor coordination is based on the diligent practice and self-cultivation. Beginners should be ready for a long-term practice, even a life long, because Health Qigong is not a crash course, which requires the accumulation of practice and the comprehension of the exercises for mental adjustment, respiratory regulation and physical adaptation over a long period of time. Only when the practitioners have a solid foundation, can they comprehend the thought and meanings of Health Qigong. Therefore, the practice of Health Qigong is an orderly process, advancing gradually from easy to difficult, from hard to skillful. To be Huo, the practitioners should be mentally calm and concentration, comprehending skillfulness, flexibility, changefulness of the movements and postures in case of disorder.

The second demand is the flexibility and coherence of the content. The practice of Health Qigong requires not only the study of the law of nature, but the actual situation and regulations of the body and soul of the practitioners themselves, trying to create the internal and external surroundings, choosing the best means, place, contents, schedule and intensity of exercises, following the natural change of Yin and Yang, while adjusting the body and mind, and eventually achieving the unity of nature and mankind.

Health Qigong has rich contents and exercises with their own characteristics. Different exercises have different characteristics, value and functions of regimens, therefore, practitioners of different conditions and demands should flexibly choose the appropriate exercises to realize their goal of regimen. Secondly, to reach a new level, the practitioners should learn and borrow the essence from other exercises or schools of Qigong, learn to apply different means and approaches as the supplement to enrich the content and draw inferences from one instance and avoid insipid practice. Thirdly, to improve the quality of the movements, it is necessary to practice some important or difficult therbligs repeatedly on purpose and deal well with the relations between practice and rest, motion and still, softness and hardness. Therefore, the practitioners should play the role of an innovator, being bold in exploration and practice, trying to apply new forms of practice.

The coherence of the content requires the practitioners' active mind and thought, especially the combination of thought, habit and practice. Practitioners can acquire the information and knowledge learned through collection, analysis, judgment, amplification and summarization, always revise the acquired knowledge, put it into practice and try to innovate and develop it by new elements, never being fettered by set patterns so that the goal of study will be realized.

\section{Dialectical Relations of Tong and Huo}

Tong and Huo is a dialectical unity, inseparably interconnected and supplementary to each other. Tong is the foundation of Huo and the guarantee of innovation and change. Only with the agility of the motions and movements, abundance of contents, the flexibility of the mind and application, from quantitative to qualitative change and the internal and external cultivation, can the sublimation of Huo and unity of form and spirit be finally realized.

Huo is the sublimation of Tong, the key to the unity of form and spirit and internal and external 
cultivation. The practitioners should cultivate internally the spirit, respiration and soul and externally the muscles, bones and skins; adapt to the nature, integrate into society and cultivate the mind, realize the unity of mankind and heaven; present one's own discipline and cultivation of regimen by appearance, gestures and speech and behavior, demonstrate the profound cultural foundation of Health Qigong.

Tong and Huo having made up the relations of dialectical unity of body and soul, mankind and nature, is a significant way of regimen and the cultural foundation of the inheritance, promotion and introduction of Health Qigong.

\section{Conclusion}

Tong and Huo, the inseparable parts and the necessity of harmony and unity, are the key elements and ways of the cultural inheritance and promotion of Health Qigong.

\section{Acknowledgments}

The study was sponsored by Sichuan Health Qigong Research Key Project, 2014, No. SCQG 2014A004 and Neijiang Normal University Research Project, 2012, No. 12NJS17, named The Current Situation and Promotion of Health Qigong.

\section{Refercences}

[1]Chinese Health Qigong Association. New Exercises of Health Qigong Series. Beijing: People’s Physical Culture Press,2010.

[7]Ji Yunxi. Health Qigong. Beijing: People’s Physical Education House, 2007.

[3]Qiu Pixiang. Chinese Traditional Regimen. Beijing: People’s Physical Culture Press,2006.

[6]Wei Yongzhong. Culture of Philosophy, Science and Qi. Changchun: Jilin University Press, 2006.

[5]Xu Wu. Developing Trend and Promotion Strategy of Health Qigong. Journal of Neijiang Normal University,2015(2):97-100.

[2]Zhang Liwen. Study of He and He. Beijing: Renmin University of China, 2006.

[4]Zheng Qin.Exercises and Culture of Tai Chi. Wuhan: Hubei People’s Press,2004. 